\title{
Monitoring of AGNs at the Shanghai Astronomical Observatory
}

\author{
B.C. Qian \& J.Tao \\ Shanghai Astronomical Observatory, Chinese Academy of Sciences, \\ Shanghai, 200030
}

\begin{abstract}
This paper briefly introduces the program, equipment and results of monitoring of AGNs carried out at Shanghai Astronomical Observatory.
\end{abstract}

\section{Introduction}

A program of monitoring of AGNs has been carried out at the Shanghai Astronomical Observatory(SHAO) since 1994. We observe more than twenty AGNs with the $1.56 \mathrm{~m}$ telescope located at She-Shan station of SHAO. The telescope is equipped with a liquid-nitrogen cooled Photometrics Series 200 CCD camera with $1024^{*} 1024$ pixels. The field of view is $4^{\prime} 17^{\prime \prime}\left(1\right.$ pixel $\left.=0.25^{\prime \prime}\right)$. A focal reducer offers a field of view of about $13^{\prime}\left(1\right.$ pixel $\left.=0.76^{\prime \prime}\right)$.

The AGN monitoring program includes two parts: participating in the International AGN Watch and monitoring of blazars. The nature of AGNs is still an open question. Photometric observations of AGNs are important in order to construct their light curves and to study their variation behavior on different time scales.

By the mid-1980s, astronomers understood that their emission-line variability was closely tied to continuum variability, which strongly supported the prevailing view that the lines were driven by photoionization from the central compact continuum source, thought to be an accretion disk surrounding a supermassive black hole. It was also recognized that the emission-line variations should follow the continuum variations, but with a time delay that reflects the light-travel time across the broad-line region (BLR).

Blazars are an extreme subclass of AGNs and often show large and violent variations. We also observed the variability of many blazars on very short time scales called intraday variability or microvariability from minutes to hours which is also a common property of blazars.

\section{Light Curves}

Light curves of AGNs will be the direct result of monitoring. Some monitoring data have already been processed and will be published in the very near future. In late autumn 1994, the International AGN Watch started a multi-wavelength monitoring campaign on 3C390.3( Dietrich et al. 1998, O'Brien et al. 1998). 


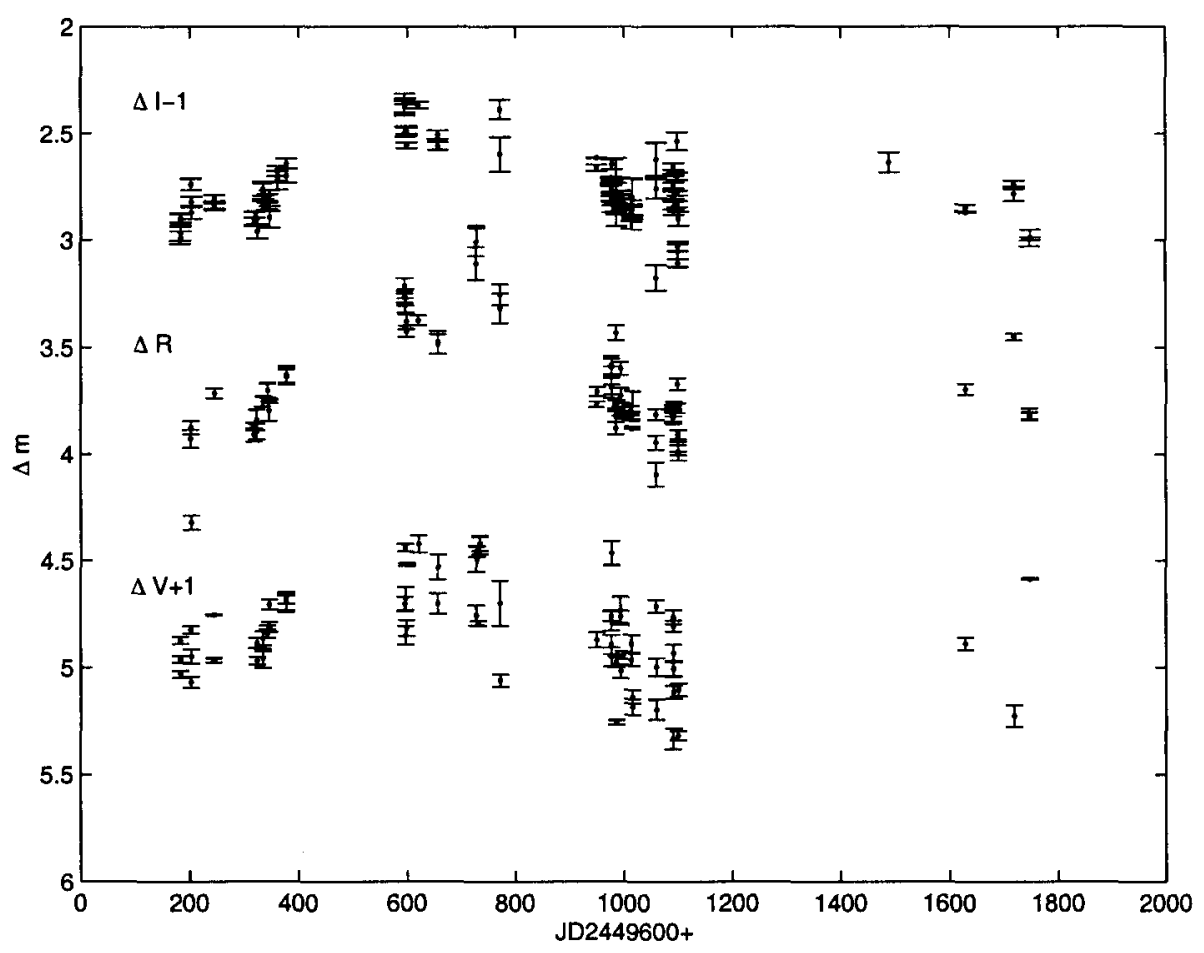

Figure 1. Light curve of $3 \mathrm{C} 390.3$ for passbands V,R and I.

Figure 1 shows the light curve of $3 \mathrm{C} 390.3$, obtained by the $1.56 \mathrm{~m}$ telescope of SHAO. It includes optical observational data of 3C390.3 up to 1999.

\section{Microvariability}

S5 $0716+714$ has exhibited significant variability. The high signal-to-noise, high time-resolution multi-frequency observations provide the most stringent constraint for physical models explaining the phenomenon of microvariability. Figure 2 shows the microvariability displayed by S5 0716+714, on March 16, 2000 . The brightness varied by about 1 mag. in two hours.

\section{The Time Lag}

Over the past ten years, considerable efforts have been made to get well-sampled light curves of AGNs through spectroscopic monitoring campaigns (Peterson 1993). The time lags of several AGNs have been determined (Peterson et al. 1998a; Peterson et al. 1998b). For the object BL Lac, Wagner et al (1996) found that no lag between $\mathrm{R}$ band and radio $(5 \mathrm{GHz}$ ) was larger than 24 hours for S5 0716+714. Nesci et al. (1998) found for BL Lac that there is no time lag between the $\mathrm{V}$ and $\mathrm{R}$ bands within an accuracy of about 15 minutes. 


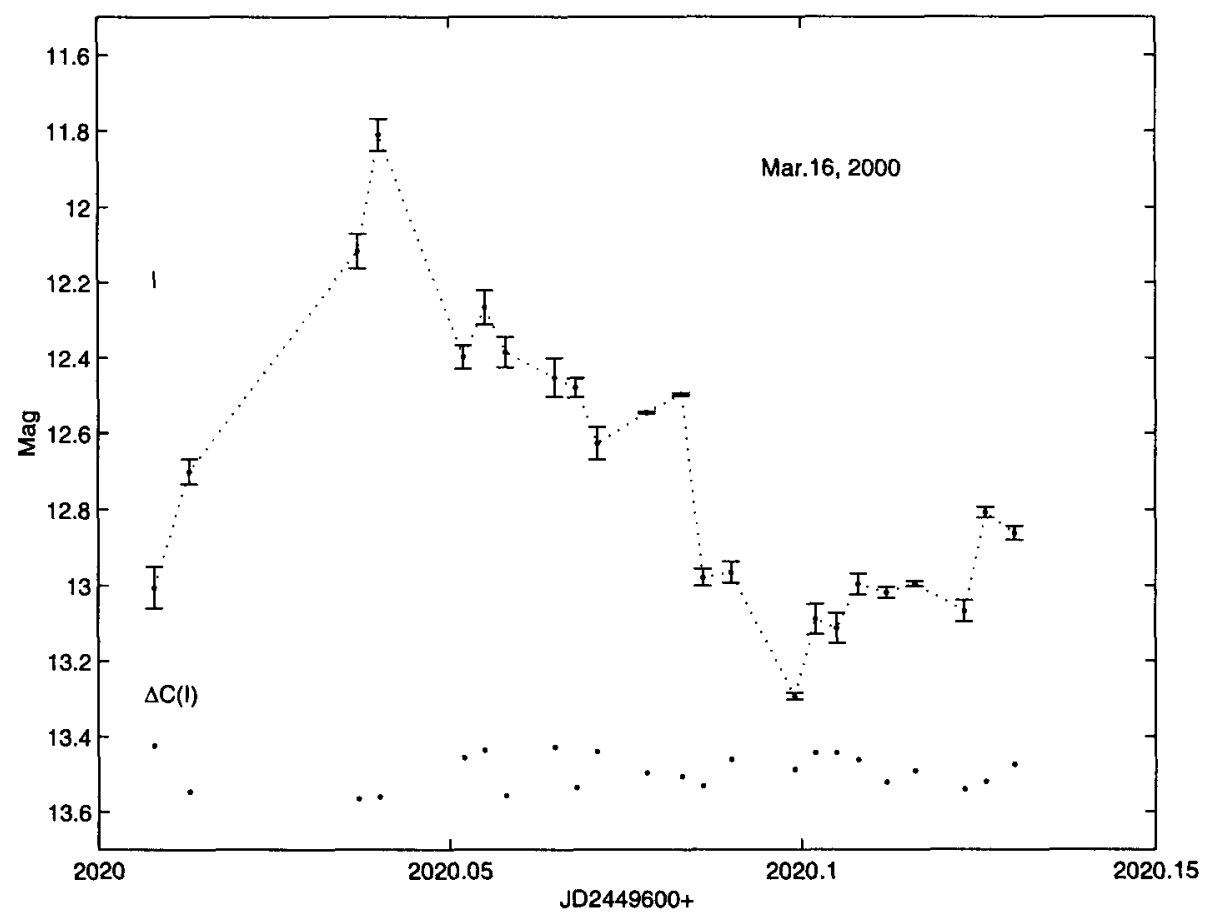

Figure 2. Light curve of S5 $0716+714$ on March 16, 2000 for passband I. The observation was taken by the $1.56 \mathrm{~m}$ telescope. The dots at the bottom are for two comparison stars. 
In monitoring of S5 $0716+714$, a whole intraday variability was detected on 1995 Jan. 8. We got 80 observations in band V and I. Using cross-correlation analysis, interpolation and Discrete Correlation Function, a six minute time lag between band V and I was obtained (Qian et. al, 2000).

\section{Quasiperiodic Oscillations}

Quasiperiodic oscillations on short time scales have been observed only in a few BL Lac objects so far. For example, for S5 0716+714, Wagner (1991) found quasiperiodic variations on time-scales of 1 and 7 days in both radio and optical wavelengths respectively. Heidt and Wagner (1996) obtained a period of 4 days for S5 0716+714. Urry et al. (1993) found quasiperiodic oscillations in PKS 2155-304 both in UV and X-ray regions on a time-scale of one day. We used the Jurkevich method (Jurkevich 1971) to search for quasiperiodic oscillations and found quasiperiodic variation on time-scales of 10 days for S5 0716+714 and BL Lac.

\section{References}

Dietrich, M. et al. 1998, ApJS 115, 185

Heidt, J. \& Wangner S.J. 1996, A\&A 305, 42

Jurkevich, I. 1971, Ap\&SS 13154

Nesci, R. et al. 1998, A\&A 332, L1

O'Brien, P.T. et al. 1998, ApJ 509, 163

Peterson, B.M. 1993, PASP 105, 247

Peterson, B.M. et al. 1998a, PASP 110, 660

Peterson, B.M. et al. 1998b, ApJ 501, 82

Qian, B. et al. 2000, PASJ 52, 1075

Urry, C.M. et al. 1993, ApJ 411, 614

Wagner, S.J. 1991, In: Duschl,W.J., Wagner, S.J., Camenzind, M.(eds.) Proc. Variability of Active Galaxies. Lecture Notes in Physics 377, SpringerVerlag, P. 163, 1991

Wagner, S.J., Witzel A., Heidt J., et al. 1996, AJ 111, 2187 